\title{
Plavec, Jan Georg: Babylon oder Blase? Die Brüsseler EU-Kommunikationskultur. Einstellungen von Politikern und Journalisten im Vergleich
}

\author{
Baden-Baden: Nomos 2020. 297 Seiten. Preis: $€ 59,00$
}

\author{
Kim Murphy
}

Angenommen: 22. Dezember 2021 / Online publiziert: 19. Januar 2022

(C) Der/die Autor(en) 2022

Trotz des allgegenwärtigen Einflusses der EU-Politik auf das Leben der EU-BürgerInnen gibt es im Bereich der politischen Kommunikation nur wenig Forschung über die Politik-Medien-Beziehungen der EU und die politische Kommunikationskultur in Brüssel. Einerseits ist die Forschung zu europäischer Öffentlichkeit reich an vergleichender Forschung, die unterschiedliche politische Kommunikations- und Journalismuskulturen in verschiedenen nationalen Kontexten untersucht, aber bislang wurde weniger untersucht, ob es im Zentrum der EU eine politische Kommunikationskultur gibt, die sich von nationalen Kulturen unterscheidet. Genau das hat der Redakteur und Wissenschaftler Jan Georg Plavec in seiner Dissertation ermittelt, die er 2020 an der Freien Universität Berlin abschloss. Im Mittelpunkt dieser relevanten Studie stehen folgende Forschungsfragen: Gibt es eine einheitliche politische Kommunikationskultur in Brüssel, die die Grundlagen der Politiker-JournalistenInteraktionen beschreibt?

Die theoretische Grundlage für die Analyse der politischen Medienarbeit der EU ist Barbara Pfetschs Konzept der politischen Kommunikationskulturen. Laut Plavec ist dies ein geeigneter konzeptioneller Rahmen, da es eine Untersuchung darüber ermöglicht, wie professionelle Einstellungen sowohl von PolitikerInnen als auch von JournalistInnen die kommunikative Interaktion zwischen beiden beeinflussen. Jan Georg Plavec leistet einen wichtigen theoretischen Beitrag, indem er das Modell von Pfetsch für die Analyse der Beziehungen zwischen Politik und Medien in der EU anpasst und aktualisiert. Sein Modell fokussiert auf eine Vielzahl von Faktoren auf der Makro-, Meso- und Mikroebene, die die Beziehungen zwischen PolitikerInnen und JournalistInnen in Brüssel potenziell beeinflussen können. Auf

Prof. Dr. Kim Murphy $(\bowtie)$

Fachbereich Journalismus und Kommunikation, Hochschule für Medien Kommunikation und Wirtschaft, Ackerstraße 76, 13355 Berlin, Deutschland

E-Mail: k.murphy@hmkw.de 
der Makroebene berücksichtigt er zum Beispiel die Tatsache, dass das europäische politische System völlig anders strukturiert ist als die nationalen politischen Systeme, in denen es in der Regel einen Staat, eine Regierung und eine Opposition gibt. All diese Kontextfaktoren sind erforderlich für das Verständnis der Einstellungen und Verhaltensweisen von JournalistInnen und PolitikerInnen auf der Mikroebene.

In seinem Literaturüberblick und seiner theoretischen Analyse beschreibt Jan Georg Plavec detailliert und prägnant das Spannungsverhältnis von JournalistInnen und PolitikerInnen in der EU wie ,between a rock and a hard place“. Zum Beispiel erklärt er die Problematik für EU-KorrespondentInnen, die auf der einen Seite versuchen, Informationen von Brüssel sachlich zu vermitteln, aber auf der anderen Seite ihre Berichte für das heimische Publikum anpassen müssen. Laut Plavec führt es zu einer Spannung zwischen nationalen und transnationalen Nachrichtenframes. Um die politische Kommunikation in der EU zu verstehen, muss man diese konkurrierenden Einflüsse (oder die Dualität) und die Rolle der transnationalen und nationalen politischen Ebene verstehen, wobei die nationale Ebene meist Vorrang hat.

In Brüssel gibt es etwa 1000 akkreditierte JournalistInnen, von denen die meisten für nationale Medienorganisationen arbeiten. Aber es gibt auch eine große Gruppe von JournalistInnen, die für transnationale Medien wie die Financial Times, den EU Observer, Euractiv und Politico arbeiten. Um die politische Kommunikationskultur der EU empirisch zu analysieren, führte Plavec 2016 eine Umfrage unter 309 JournalistInnen nationaler und internationaler Medien sowie PolitikerInnen aus Rat, Kommission und Parlament der EU durch. In seiner Umfrage untersuchte er die politischen Medienbeziehungen anhand von vier Dimensionen: Input (z. B. Bürgerbefragungen, LobbyistInnen), System (z. B. Wahrnehmung des Medieneinflusses und der Medienmacht), Output (z. B. Framing in der Berichterstattung) und Rollendimensionen (Einstellung der Akteure zu ihren Rollen).

$\mathrm{Zu}$ den aufschlussreichsten Ergebnissen gehörte, wie viel Einfluss LobbyistInnen im Vergleich zu Bürgern auf die Berichterstattung in den Medien haben. Er untersuchte, inwieweit JournalistInnen Kontakte zu LobbyistInnen in Brüssel haben, und stellte fest, dass der stärkste Kontakt zwischen internationalen LobbyistInnen und JournalistInnen besteht, die für transnationale Elitemedien wie die Financial Times arbeiten. Die durchschnittliche Zahl der Kontakte zwischen JournalistInnen und internationalen LobbyistInnen war sogar höher als die Zahl der Kontakte zwischen JournalistInnen und PolitikerInnen. Am beunruhigendsten ist jedoch, dass Meinungsumfragen bei der Beeinflussung der Medienberichterstattung kaum eine Rolle spielen.

Er hat auch ermittelt, inwieweit die Befragten nationales Framing politischer Botschaften als eine effektive Output-Strategie sehen. Wie erwartet haben die meisten Interviewten zugestimmt, dass es effektiv sei, EU-politische Kommunikation national zu framen. Gleichzeitig stimmten die meisten Befragten auch zu, dass eine angemessene EU-Kommunikation voraussetze, dass JournalistInnen auch die politischen Positionen anderer Länder widerspiegelten und nicht einen engen nationalen Rahmen vorgeben sollten. Dieser Widerspruch wurde von der Mehrheit der Befragten festgestellt und zeigt ,eine negative bis zynische Haltung der Brüsseler Kommunikationseliten vor allem gegenüber nationalen Publika“" (S. 188), die ihrer Meinung 
nach nicht in der Lage sind, die EU-Politik ohne diese nationalen Frames zu verstehen. Obwohl es viele interessante Ergebnisse gab, verdeutlichen diese beiden Ergebnisse, wie die derzeitige EU-Kommunikationskultur zum Demokratiedefizit in Europa beiträgt.

Obwohl diese Studie einen wichtigen Beitrag zum Bereich der politischen Kommunikation und zu unserem Verständnis der politischen Medienarbeit in der EU leistet, gab es einige wichtige Aspekte der politischen Kommunikation von heute, die der Autor nicht untersucht hat. Zum Beispiel betonten die Befragten den Einfluss der sozialen Medien auf die Politik, aber es gab keine weiteren Fragen in der Studie zu deren Auswirkungen. Ein weiterer Aspekt, der in dieser Studie fehlte, waren einige sehr wichtige, kontextbezogene Faktoren. Zum Beispiel wurde die Umfrage 2016 durchgeführt, ein Jahr nach der Flüchtlingskrise in Europa und im selben Jahr wie das Brexit-Referendum in Großbritannien. Dennoch gab es in dem Buch keinen Überblick über diese wichtigen politischen Ereignisse und darüber, wie sich diese auf die Beziehungen zwischen Medien und Politik in Brüssel ausgewirkt haben könnten. Nichtsdestotrotz ist diese Studie insgesamt ein sehr wichtiger Beitrag zu diesem Thema.

Funding Open Access funding enabled and organized by Projekt DEAL.

Open Access Dieser Artikel wird unter der Creative Commons Namensnennung 4.0 International Lizenz veröffentlicht, welche die Nutzung, Vervielfältigung, Bearbeitung, Verbreitung und Wiedergabe in jeglichem Medium und Format erlaubt, sofern Sie den/die ursprünglichen Autor(en) und die Quelle ordnungsgemäß nennen, einen Link zur Creative Commons Lizenz beifügen und angeben, ob Änderungen vorgenommen wurden.

Die in diesem Artikel enthaltenen Bilder und sonstiges Drittmaterial unterliegen ebenfalls der genannten Creative Commons Lizenz, sofern sich aus der Abbildungslegende nichts anderes ergibt. Sofern das betreffende Material nicht unter der genannten Creative Commons Lizenz steht und die betreffende Handlung nicht nach gesetzlichen Vorschriften erlaubt ist, ist für die oben aufgeführten Weiterverwendungen des Materials die Einwilligung des jeweiligen Rechteinhabers einzuholen.

Weitere Details zur Lizenz entnehmen Sie bitte der Lizenzinformation auf http://creativecommons.org/ licenses/by/4.0/deed.de.

Prof. Dr. Kim Murphy ist Professorin für Unternehmenskommunikation und Fachbereichsleiterin des Fachbereichs Journalismus und Kommunikation an der Hochschule für Medien, Kommunikation und Wirtschaft Berlin. 